\title{
A MAINTENANCE POLICY SELECTION TOOL FOR INDUSTRIAL MACHINE PARTS
}

Jean Khalil ${ }^{1}$, Sameh M Saad ${ }^{2}$, Nabil Gindy ${ }^{3}$, Ken MacKechnie ${ }^{4}$

1- School of Mechanical, Materials, Manufacturing Engineering and Operations

Management, The University of Nottingham.epximbk@nottingham.ac.uk

2- School of Engineering, Sheffield Hallam University, City Campus, Sheffield,

S11WB, UK, s.saad@shu.ac.uk

3- School of Mechanical, Materials, Manufacturing Engineering and Operations Management, The University of Nottingham. Nabil.Gindy@nottingham.ac.uk 4- Rolls-Royce PLC, Plant MTM Leader, Fan Systems.

\begin{abstract}
Industrial maintenance activities may be categorised under three strategies, preventive, corrective and predictive. It is necessary to identify domains of equipment and decide which maintenance policy suits each domain of equipment. Usually, it is assumed that maintenance managers are capable of achieving this job. This assumption however is practical, relies on the human factor, which as known to humans, could involve mistakes and hence may lead to implementing the wrong maintenance strategies. Money losses would eventually result. This paper presents a tool that deals with equipment as machine parts domains, where the domain is the group of the similar machine parts which undergo the same conditions. The suggested tool consists of a dual criteria categorisation grid, which through historical data, experts' knowledge and mathematical formulation selects the most suitable maintenance policy for each machine part (domain) individually. The implementation of this tool should guarantee the execution of the appropriate maintenance policy with each and every machine part; therefore it should result in a more economical production function and a more efficient maintenance function. Examples from industry are given to further clarify the proposed tool applications.
\end{abstract}

\section{INTRODUCTION AND BACKGROUND}

Equipment maintenance is a key contributor to the welfare of a production organisation. The optimisation of maintenance cost is the focus of many research works. But, before one can develop a maintenance cost optimisation model, two important questions should be answered:

- Which maintenance policy best fits the application?

- What is the level of the application; by mean would the whole industrial site adopt one maintenance policy; May each group of machines adopt a maintenance policy that suits it; or should the problem be studied on the more detailed ground of the machine parts? 
(Wang 2002) produced a survey of the literature discussing maintenance policies. At the end some remarks were given about the optimal maintenance policy. (Chiang and Yuan 2000) and (Moustafa et al 2004) studied the deterioration of a system using a Markov chain and a semi Markov chain respectively, in order to select the best maintenance policy from: do nothing, repair and replace. Both models are discussing maintenance actions rather than maintenance policies. Their work related more to the response of the maintenance department to the life's evolution rather than the pre-planning of the optimum maintenance policy. (Bevilacqua and Braglia 2000) used the Analytical Hierarchy Process to select the optimum maintenance policy, they considered five strategies, they categorised a firm's equipment into three groups based on criticality, a nd hence a ppointed a policy to each group of machines. (Wang 2003) categorised maintenance policies into

1- scheduled

2- preventive (time periodical)

3- Condition based (C.B.M)

Fuzzy logic was then implemented to develop a model that would assist the maintenance manager in selecting the best maintenance strategy. For C.B.M the model would also assist in choosing the most suitable technique.

One common feature between the surveyed approaches is that they tackle whole machines; however a key point of the tool suggested in this paper is that it tackles machine parts. Therefore it takes the study to a more detailed level; this aspect of the proposed tool allows the flexibility of appointing different maintenance policies to different parts of the same machine. Clearly this flexibility will lead to better results in terms of the effectiveness and efficiency of the maintenance activities.

Figure 1 shows a decision making grid proposed by (Labib et al 1998) developed to recommend maintenance decisions (for machines) to management. "This grid acts as a map, where the performances of the worst machines are placed based on multiple criteria. The objective is to implement appropriate actions that will lead to the movement of machines towards the northwest section of low downtime, and low frequency. In the top left region, the action to implement, or the rule that applies, is OTF (operate to failure). The rule that applies for the bottom-left region is SLU (skill level upgrade) because data from breakdowns - attended by maintenance engineers - indicate that machine $[G]$ has been visited many times (high frequency) for limited periods (low downtimes). In other words maintaining this machine is a relatively easy task that can be passed to operators after upgrading their skill levels. A machine that is located in the top-right region, such as machine [B], is a problematic machine, in maintenance words "a killer". It doesn't break down frequently (low frequency), but when it stops it is usually a big problem that lasts for a long time (high downtime). In this case the appropriate action to take is to analyse the breakdown events and closely monitor its condition, i.e.: condition based monitoring (CBM). A machine that enters the bottom right region is considered to be one of the worst performing machines based on both criteria. It is a machine that, maintenance engineers are used to seeing not working rather than performing normal operating duty. A machine of this category, such as machine [C], will need to be structurally modified and major design-out projects need to be considered, and hence appropriate rule to implement the design out maintenance (DOM). 
If one of the antecedents is a medium downtime or a medium frequency, then the rule to apply is to carry on with the preventive maintenance schedules" Labib et al 1998.

The aim of this paper is to develop a classification grid tool, in order to assist the maintenance manager in appointing the right maintenance policy to every machine part. The suggested tool uses experts' information i.e. from the industrial site subject to the study, in order to assist the maintenance manager in making decisions, usually found gloomy and confusing.

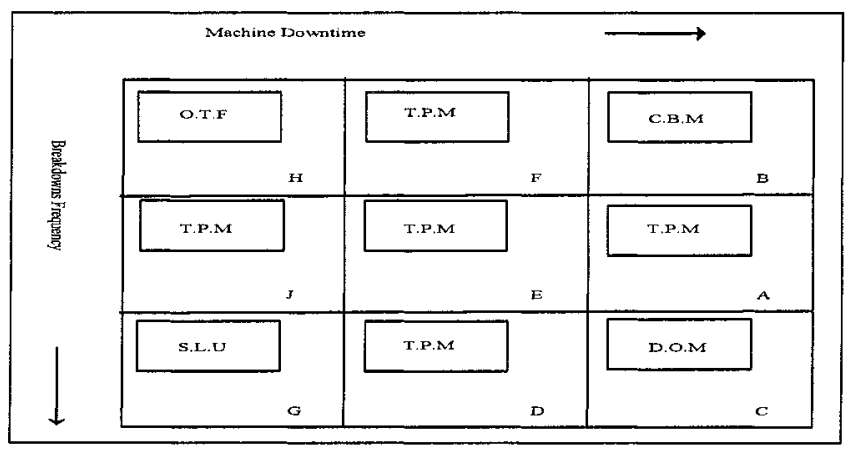

Figure 1- Factory Improvement Grid (Labib et al 1998)

\section{2- RESEARCH METHODOLOGY AND PROPOSED GRID}

The improvement grid as explained in previous section, aims to categorise a firm's machines with respect to two criteria: total downtime and breakdown frequency, in order to implement the right maintenance policy with every category of machines. The suggested improvement grid is modified to suit the application tackled by this paper as follows:

Instead of categorising a firm's machines; machine parts are categorised, in order to decide which policy would best fit every group of machine parts. This fact would allow different parts of the same machine to undergo different maintenance policies as appropriate. That feature, on its own should lead to significant machinehealth improvement as each of its components will undergo the appropriate maintenance policy, rather than having to undergo the general policy that is labelled to the whole machine.

In Labib's paper, the judgements are called relative judgements. Relative judgements are the opposite of absolute judgements. For example, based on relative judgements, a school director could decide to gather the scientifically worst five pupils of every class for a meeting. Whereas based on absolute judgements, the director would decide to gather all the pupils who failed a certain examination. The difference is, that in the first case the pupils are judged in comparison with others while in the second case the students are judged with respect to an absolute scale (i.e. exam marks), against which every student is assessed individually. Within the tool suggested in this paper absolute judgements are implemented because they are believed to suit better this application.

Absolute judgements allow the experts to enter critical values rather than 
assuming them to be a proportion of the maximum available value. Also absolute judgement can accommodate new candidates i.e. machine parts, at any time, whereas within relative judgement the whole population i.e. ALL the machine parts must be considered right at the beginning. If one more machine part was to be added at any point of time, the whole process has to be reset.

Machine parts are assessed with respect to two criteria: the failure frequency and the cost of failure inflected on the industrial site at the event of failure. The downtime considered in Labib's grid will be one of the factors composing the cost of failure criterion. In the following sections the two criteria will be discussed.

\subsection{The failure frequency criterion}

The failure frequency criterion reflects the repetitiveness of enquiries the machine part causes to the maintenance department in an industrial firm. It expresses the load it puts on maintenance people in terms of number of jobs dispatched per unit of time. For them a job no matter how small, adds to the queue and needs response within a given time. The capture of this information should be a straight forward function of any maintenance data management system. It is only needed to know the number of failures of the given machine part per a given period of time, then divide the number of failures per the number of time units and thus get a frequency; for example if it was found that through the last six month, a certain part was reported 36 times, then the frequency is 6 failures/month. The time unit (month) is not critical, it could be a quarter or a week, the critical aspect is to compare all parts on the same basis, therefore calculate all the frequencies using the same units and plot them on the same grid. Despite the significance of the failure frequency criterion, it does not show the impact of a given failure, which could affect not only the maintenance department but many other parties. It is thought that the best way of reflecting the consequences of a failure is by estimating the financial losses it causes. The cost of failure criterion calculates these losses.

\subsection{The Cost of failure criterion}

Expressing the most considerable financial impacts of a failure resembles to a scoring technique, but in this case instead of using non-meaningful digits, currency is used. The usage of currency as a score allows the user to better feel the meaning of the output. The "cost of failure" criterion includes in its calculations four factors, crucial to the assessment of the criticality of a failure. These criteria are bottleneck penalty, production lost opportunities, possible effect of the fault on scraping production and the waste of resources in terms of labour. The price of the spare part is not included within the calculation of the cost of failure. The reason is that this criterion aims at reflecting the financial impact of the machine part's failure on the industrial site, irrespective of its own price. Had the spare part price being included, misleading calculations would have resulted.

The calculations of the most relevant cost factors that result at a failure event are as follows: 
$\begin{array}{ll}\text { Production } \\ \text { losses cost }\end{array} \quad L_{p}= \begin{cases}\left(\mathrm{t}_{\mathrm{ti}}+\mathrm{t}_{\mathrm{su}}\right) \times \frac{\alpha}{\pi} & \text { at tsu }>0 \\ \frac{\mathrm{t}_{\mathrm{ti}}}{\pi} \times \alpha & \text { otherwise }\end{cases}$

ii-Products $\quad L_{d}=v \times c_{p d}$

damage cost

iii-

Bottleneck

penalty cost

$$
L_{b}= \begin{cases}\left(t_{t i}+t_{s u}\right) \times c_{d p} & \text { at } t s u>0 \\ t_{t i} \times c_{d p} & \text { otherwise }\end{cases}
$$

iv- The

$$
B_{l}=
$$

booked

labour cost

$$
\left\{\begin{array}{l}
\left.\mathrm{x}_{1} \times \mathrm{t}_{\mathrm{lc}} \times \mathrm{s}_{\mathrm{mh}}+\mathrm{x}_{2} \times\left(\mathrm{t}_{\mathrm{ti}}+\mathrm{t}_{\mathrm{su}}\right) \times \mathrm{s}_{\mathrm{oh}}\right) \text { at } t_{\mathrm{su}}>0 \\
\mathrm{x}_{1} \times \mathrm{t}_{\mathrm{lc}} \times \mathrm{s}_{\mathrm{mh}}+\mathrm{x}_{2} \times \mathrm{t}_{\mathrm{ti}} \times \mathrm{s}_{\mathrm{oh}} \quad \text { otherwise }
\end{array}\right.
$$

The summation of these factors forms the expression presented in equation 5 $C_{\text {failure }}=$

$$
\left.\left[\left(t_{i i}+t_{s u}\right) \times \frac{\alpha}{\pi}+v \times c_{p d}+\left(t_{t i}+t_{s u}\right) \times \frac{c_{d p}}{\pi}+x_{l} \times t_{l c} \times s_{m h}+x_{2} \times\left(t_{t i}+t_{s w}\right) \times s_{o h}\right)\right]
$$

Table 1 shows the definition of the above mentioned symbols. Due to space limitations these symbols will be explained in details during the conference presentation.

Table 1- Variables composing the cost of failure function

\begin{tabular}{|l|l|l|}
\hline Symbol & Definition & Unit \\
\hline$c_{d p}$ & Cost of production-line delay per unit of time. & $f / \mathrm{h}$ \\
\hline$c_{p d}$ & Value of one damaged work-piece. & $£$ \\
\hline$s_{m h}$ & Maintenance personnel hourly rate. & $\mathrm{f} / \mathrm{h}$ \\
\hline$s_{o h}$ & Operator's hourly rate. & $f / \mathrm{h}$ \\
\hline$t_{l c}$ & $\begin{array}{l}\text { The time spent by maintenance personnel in fixing problems in } \\
\text { case of corrective action. }\end{array}$ & $\mathrm{h}$ \\
\hline$t_{t i}$ & Production time loss due to a fault excluding time of set up. & $\mathrm{h}$ \\
\hline$t_{s u}$ & Machine set up time & $\mathrm{h}$ \\
\hline$v$ & Number of damaged work-pieces because of a failure. & \\
\hline$x_{l}$ & $\begin{array}{l}\text { the number of maintenance personnel involved in the repair } \\
\text { action }\end{array}$ & \\
\hline$x_{2}$ & The number of operators made on stand by because of a failure & \\
\hline$\alpha$ & Department's income due to the production of one work-piece & $f$ \\
\hline$\pi$ & A product cycle time. & $\mathrm{h}$ \\
\hline
\end{tabular}




\section{3- MACHINE PARTS CATEGORISATION GRID}

The machine parts categorisation grid is a two axes plot (see figure 2)

\subsection{First Axis, machine part failure frequency criterion (MPF frequency).}

On the failure frequency axis, the user appoints a critical value which may be defined as:

A frequency of machine parts failure occurrences that the user considers high enough, to draw the attention, for improvement efforts.

\subsection{Second Axis, machine part failure cost (MPF cost)}

On the cost of failure axis the user allocates two critical values: a lower cost of failure value (CLCV), and an Upper cost of failure value (CUCV). The lower value is the maximum cost of a failure that would be considered insignificant and cheaper than the cost of an operator training course. The upper value is the maximum cost of a failure that would be cheaper to repair by the operator.

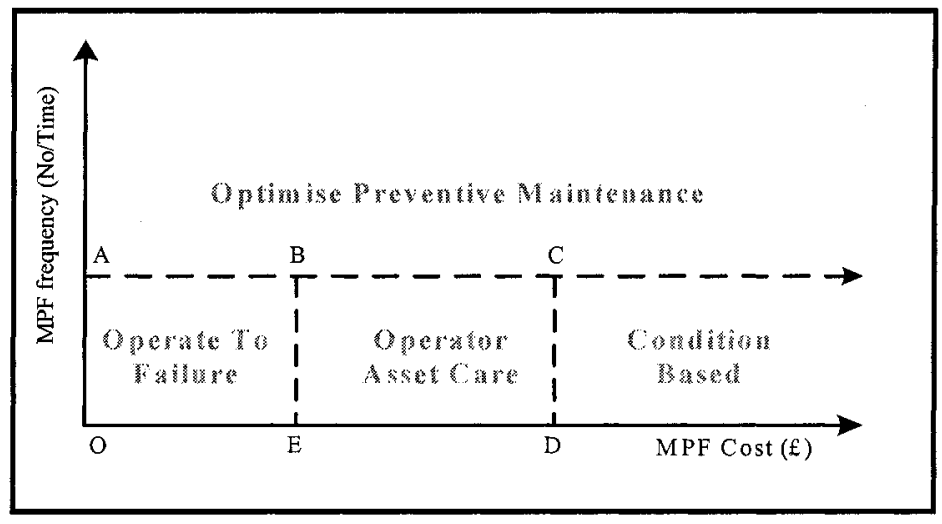

Figure 2- Modified Optimisation Grid

At this point the user should have a two axes plot with the Failure frequency criterion on one axis and the Cost of failure criterion on the other, and with one critical value for the failure frequency criterion and two critical values for the cost of failure criterion (see figure 2). The user should then start allocating the factory's machine parts subject to the study.

The factory's machine $\mathrm{p}$ arts will therefore be a llocated on the $\mathrm{c}$ ategorisation grid (see figure2) as follows:

1. A group of machine parts may fall in rectangle (OABE), this group should undergo and operate to failure policy, and hence they will run until they fail on which case a corrective action will take place.

2. Another group of machine parts may fall in rectangle (EBCD), this group should undergo an operator asset care, by mean the machine operator should be trained to repair the failure of these particular machine parts.

3. A third group will have a higher failure cost than D and a lower failure 
frequency than $\mathrm{A}$. The suitable maintenance policy for this group is condition based maintenance, achieved through condition monitoring or routine inspections.

4. The fourth group will have a higher failure frequency than A. For this group periodical preventive maintenance (e.g. time based) is the best policy. The usage of a preventive maintenance model is recommended with this group of machine parts.

A preventive maintenance model aiming at optimising the total cost of maintenance for machine $p$ arts was developed in (Khalil et a 12003 ). The application of the model r eturns the cost-optimum life span of a machine part; hence a preventive maintenance action could take place and therefore avoid the occurrence of a failure. In other words, the outcome of this model assists the decision taker in the best timing with respect to cost for preventive maintenance actions. The tool proposed in this paper may be implemented prior to the application of that model or as a stand alone tool.

The operator a sset $\mathrm{c}$ are mentioned with group 2 is o ne of the a ssumptions of the T.P.M first developed by the Japanese industry. T.P.M consists briefly of involving the operator in taking care of the machine health. Within a T.P.M. policy, beside routine tasks (oiling and lubricating) the operator could also be asked to complete the repair of some faults. Usually, the list of faults that may be repaired by the operator grows up gradually. The old behaviour "this is not my job" gets replaced by "I should better take care of $\boldsymbol{m y}$ equipment". But this transfer can't happen instantaneously. Actually educating the operators could be a hard task that needs time and effort. T.P.M proved in many cases to be successful and efficient however some firms prefer to adopt a different policy separating between the operators' duties and the service people duties; they thus prohibit to the operator the involvement in any equipment technical action. In industry, the latter policy is usually not particularly appreciated by the operators, as it leads to time-losses; however it is usually advocated to be safer for the operators and better for the equipment, because it only allows skilled people to work on the equipment.

\section{EXAMPLES FROM INDUSTRY}

The following studies were carried at a multinational industrial organisation specialised in aero-industry. Four machine parts subject to the study would be referred at, as machine part A, B, C and D. Frequency critical value is 4 failures/month, Cost lower critical value: CLCV is $£ 300$ and Cost upper critical value is CUCV: $£ 1000$. Figure 3 displays the grid for these examples and table 1 shows the collected data for these four parts. 


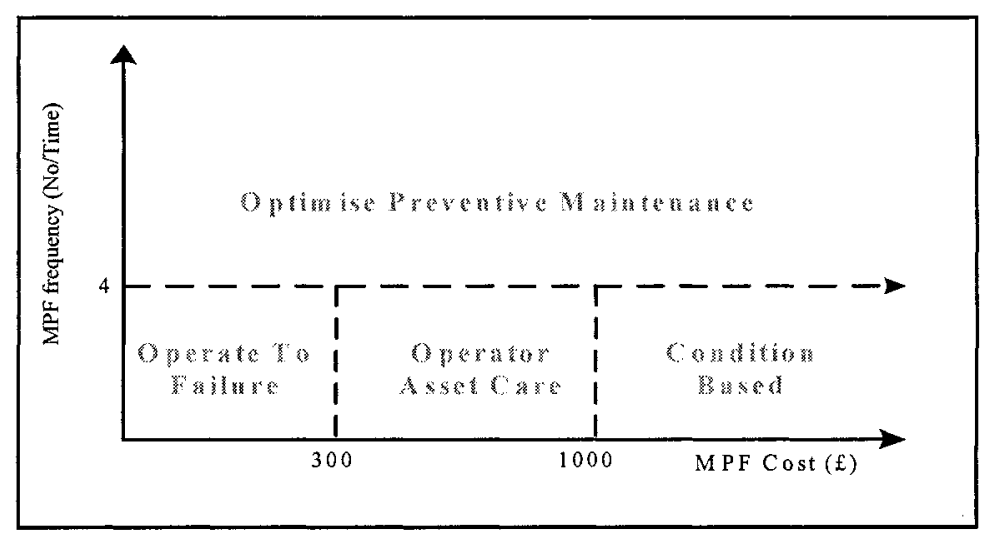

Figure 3- Illustrative Example Grid

Table 2- Real Values of the machine parts variables

\begin{tabular}{|l|c|c|c|c|}
\hline Abbreviation & \multicolumn{4}{|c|}{ Unit } \\
\hline & Part A & Part B & Part C & Part D \\
\hline$c_{d p}$ & $£ 200$ & $£ 500$ & $£ 200$ & $£ 150$ \\
\hline$c_{p d}$ & $£ 900$ & $£ 0$ & $£ 650$ & $£ 0$ \\
\hline$s_{m h}$ & $£ / \mathrm{h} 25$ & $25 £ / \mathrm{h}$ & $£ / \mathrm{h} 25$ & $£ / \mathrm{h} 25$ \\
\hline$s_{o h}$ & $£ / \mathrm{h} 25$ & $25 £ / \mathrm{h}$ & $£ / \mathrm{h} 25$ & $£ / \mathrm{h} 25$ \\
\hline$t_{l c}$ & $4 \mathrm{~h}$ & $6 \mathrm{~h}$ & $1.5 \mathrm{~h}$ & $6 \mathrm{~h}$ \\
\hline$t_{l p}$ & $0.5 \mathrm{~h}$ & $4 \mathrm{~h}$ & $1 \mathrm{~h}$ & $4 \mathrm{~h}$ \\
\hline$t_{t i}$ & $8 \mathrm{~h}$ & $6 \mathrm{~h}$ & $4 \mathrm{~h}$ & $6 \mathrm{~h}$ \\
\hline$t_{s u}$ & $4 \mathrm{~h}$ & $0 \mathrm{~h}$ & $0 \mathrm{~h}$ & $0 \mathrm{~h}$ \\
\hline$v$ & 1 & 0 & 1 & 0 \\
\hline$x_{1}$ & 2 & 1 & 1 & 2 \\
\hline$x_{2}$ & 1 & 1 & 1 & 1 \\
\hline$\alpha$ & $£ 250$ & $£ 320$ & $£ 150$ & $£ 50$ \\
\hline$\pi$ & $1 \mathrm{~h}$ & $1.5 \mathrm{~h}$ & $1.5 \mathrm{~h}$ & $0.5 \mathrm{~h}$ \\
\hline
\end{tabular}

\subsection{Case 1: Machine part A}

By substitution in equation 5 ,

$\left.C_{\text {failure }}=[(8+4) \times 250+900+(8+4) \times 200+2 \times 4 \times 25+(8+4) \times 25)\right]=\$ 6800$

The average failure frequency of this machine part was retrieved from the data management system and found to be $\mathbf{5 . 5}$ failure/ month. Therefore, PM is recommended.

\subsection{Machine part B}

By substitution in equation 5 , 


$$
\mathrm{C}_{\text {failure }}=6 \times \frac{320}{1.5}+6 \times \frac{500}{1.5}+6 \times 25+(6 \times 25)=£ 2630
$$

The average failure frequency of this machine part was retrieved from the data management system and found to be $\mathbf{1 . 4 1}$ failures / month. Therefore, condition based maintenance is recommended.

\subsection{Machine part $\mathrm{C}$}

By substitution in equation 5 ,

$$
\mathrm{C}_{\text {failure }}=4 \times \frac{150}{1.5}+650+4 \times \frac{200}{1.5}+1.5 \times 25+4 \times 25=£ \mathbf{1 7 2 0 . 5}
$$

The average failure frequency of this machine part was retrieved from the data management system and found to be $\mathbf{7 . 2}$ failure/ month. Therefore, PM is recommended.

\section{4: Machine part $D$}

By substitution in equation 5 ,

$\left.C_{\text {failure }}=\left[6 \times \frac{50}{0.5}+0+(6) \times \frac{150}{0.5}+2 \times 6 \times 25+6 \times 25\right)\right]=\mathbf{£ 2 8 5 0}$

The average failure frequency of this machine part was retrieved from the data management system and found to be $\mathbf{1 . 1}$ failure/ month. Therefore, Condition Based maintenance is recommended.

\section{DISCUSSION}

- One important aspect of this work is that it allows the individual consideration of each part within the machine. The previous approaches that consider the machine as the unit of the study undermine the fact that, the behaviours of the parts is usually unrelated.

- Within the calculations of the cost of failure, had the cost of the spare part been included, it would have been impossible to consider different machine parts under the same grid because $C_{\text {failure }}$ critical value could not then apply. The reason is: the machine parts' own values, would manipulate the cost of failure rather than allowing it to purely reflect the impact of its failure on the firm.

- The idea of classification and arrays is not new in maintenance science however, up to the authors' knowledge it was never used to categorise machine parts. The maintenance problem was merely ceased in a bottom up technique and thus machine parts were most of the time out of the researchers' focus. 
- The development of this tool integrates to the value of preventive maintenance model presented in (Khalil at al 2003) as a decision support system, the reason being it takes away one source of potential human error; in other words substitute one human decision by a scientifically based decision. Even though the human factor contributes to the implementation of this tool, the risk of error is incredibly less than a pure human selection.

- By implementing this tool, the level of expertise required for the usage of the model presented in (Khalil et al 2003) has become less than before, which makes it more practical. After this work, the user only needs to set the critical values for both criteria (Total cost of failure and frequency of failures).

- The simplicity of the idea and its straight application should make it welcomed in industry. This paper could be taken as a good example to prove that significance and simplicity are not contradictory. It is sometimes wrongly believed that when it comes to research in industry, a limit of simplicity should be respected in order for the work to be significant.

\section{CONCLUSION}

This paper presented a tool aiming to assist the maintenance manager in selecting the most suitable maintenance policy for machine parts. Expert judgements are used to $b$ uild a $t$ wo $p$ arameters $c$ ategorisation grid on which, a firm machine $p$ arts a re categorised into four categories. Four maintenance strategies are considered to meet the nature of the four categories of machine parts. The suggested tool may be integrated to the previously developed preventive maintenance model developed by Khalil et al 2003 as a suitability test in which case, it eases up the usage of the model and makes it possible for a wider range of people. It also could be implemented as a stand alone tool.

\section{REFERENCES}

1. Bevilacqua, $M$ and Braglia, $M$. The analytic hierarchy process applied to maintenance policy selection. Reliability Engineering and System safety 2000; 70: 71-83.

2. Chinag JH and Yuan J. Optimal maintenance policy for Markovian system under periodic inspection. Reliability Engineering and System safety 71 2001; 71: 165-172.

3. Khalil J, Saad S and Gindy N. A cost optimisation decision support model for preventive and corrective maintenance actions. Proceeding of the International Conference on flexible automation and intelligent manufacturing. FAIM 2003, Florida, USA.

4. Labib AW. World-class maintenance using a computerised maintenance management system. Journal of Quality in Maintenance Engineering, 1998, Vol. 4 No. 1: pp. 66-75.

5. Moustafa MS Abdel Maksoud EY Sadek S. Optimal major and minimal maintenance policies for deteriorating systems. Reliability Engineering and System safety. 2004; 83: 363-368.

6. Mechefske CK. Wang Z. Using fuzzy linguistic to select optimum maintenance and condition monitoring strategies. Mechanical Systems and Signal Processing 2003; 17(2): 305-316.

7. Wang $\mathrm{H}$. A survey of maintenance policies of deteriorating systems. European journal of operational research. 2002, 139: 469-489. 